\title{
A criterion tor differentiabilify
}

By A. M. Macbeath.

There are two alternative methods of defining the concept of "convergence" of a sequence, one involving explicit mention of the limit, the other (Cauchy's condition) giving a necessary and sufficient condition in terms of the elements of the sequence only. The two definitions are equivalent, because of the property of completeness of the real number system.

In a similar way, the standard definition of differentiability of a function of $n$ variables makes explicit mention of the partial derivatives. It is natural to ask whether it is possible, as in the case of sequences, to give a necessary and sufficient condition which does not involve the partial derivatives at all. In this note, we show that it is possible.

Some guide to the form of the final answer is obtained by considering the case of one variable. Then differentiability means the existence of a limit, and the result, which is a special case of Cauchy's condition, is as follows:

Let $f(x)$ be a function of one real variable, $f(0)=0$. Then a necessary and sufficient condition for $f(x)$ to be differentiable at $x=0$ is the truth of the following assertion:

Given $\epsilon>0$, there exists a number $\delta>0$, such that, if $|x|,|y|<\delta$ then

$$
\left|\frac{f(x)}{x}-\frac{f(y)}{y}\right|<\epsilon .
$$

It might seem at first sight too much to expect a similar simple criterion to exist for functions of more than one variable; for the existence of partial derivatives as limits is no guarantee that the function is differentiable. However, the condition can be generalized if $(1)$ is rewritten in the determinantal form:

$$
\left\|\begin{array}{cc}
f(x) & f(y) \\
x & y
\end{array}\right\| \leqslant \epsilon|x||y| \text {. }
$$

The conditions can then be expressed, less accurately, but more "intuitively," in the form:

" $f(x)$ is approximately a linear function of $x$ (i.e. is differentiable) if the determinant on the left of (2) approximately vanishes."

We shall see that, in this form, the condition generalizes immediately. In dealing with functions of $n$ variables a single letter $x$ is used 
to denote the vector, or set of $n$ variables, $\left(x^{1}, \ldots, x^{n}\right)$. Indices are used to distinguish co-ordinates, and suffixes to distinguish different vectors. Thus the vectors $x_{1}, x_{2}$ have co-ordinates $x_{1}{ }^{i}(i=1, \ldots, n)$, $x_{2}{ }^{i}(i=1, \ldots, n)$ respectively. The symbol $|x|$ is used to denote the absolute value $\left\{\Sigma\left(x^{i}\right)^{2}\right\}^{\frac{1}{2}}$.

A function $f(x)$, with $f(0)=0$ is said to be differentiable at $x=0$ if there is a linear function $l(x)=p_{1} x^{1}+\ldots+p_{n} x^{n}$ with the property that

$$
f(x)=l(x)+o(|x|),
$$

or, more precisely, if, for every $\epsilon>0$, there exists $\delta>0$ such that, when $|x|<\delta$,

$$
|f(x)-l(x)|<\epsilon|x|
$$

Differentiability in general can be defined in terms of this special case; for $Q(x)$ is differentiable at $x=a$ if $f(x)=Q(a+x)-Q(a)$ is differentiable at $x=0$. We restrict ourselves, therefore, to the case $a=0$, $f(a)=0$.

We can now state the general theorem. Let $x_{1}, \ldots, x_{n+1}$ be any $n+1$ rectors, their components making up $n(n+1)$ variables in all. Let $f(x)$ be any function of $n$ variables. Define $D\left(f ; x_{1}, \ldots, x_{n+1}\right)$ to be the $(n+1) \times(n+1)$ determinant whose $r^{\text {th }}$ row is

$$
f\left(x_{r}\right), x_{r}{ }^{1}, \ldots, x_{r}{ }^{n} \text {. }
$$

THeOREM. Suppose that $f(0)=0$.

Then the following condition is necessary and sufficient for $f(x)$ to be differentiable at $x=0$ :

Given $\epsilon>0$, there exists $\delta>0$ such that, if $\max \left[\left|x_{1}\right|, \ldots,\left|x_{n+1}\right|\right]<\delta$, then

$$
\left|D\left(f ; x_{1}, \ldots, x_{n+1}\right)\right|<\epsilon\left|x_{1}\right| \ldots\left|x_{n+1}\right| \text {. }
$$

Proof.

A. Necessity.

Suppose that $f(x)$ is differentiable and let $p_{1}, \ldots, p_{n}$ be the partial derivatives. Define $y_{1}, \ldots, y_{n+1}$ by the equations

$$
f\left(x_{i}\right)=p_{1} x_{i}{ }^{1}+\ldots+p_{n} x_{i}{ }^{n}+y_{i} .
$$

Let $q_{1}, \ldots, q_{n+1}$ be the co-factors of $f\left(x_{1}\right), \ldots, f\left(x_{n+1}\right)$ in the determinant $D$. We deduce from (3), multiplying the equations by $q_{i}$ and adding, that

$$
D\left(f ; x_{1}, \ldots, x_{n+1}\right)=\Sigma y_{i} q_{i} .
$$

By a theorem of Hadamard ${ }^{1}, \quad\left|q_{i}\right| \leqslant \prod_{j \neq i}\left|x_{j}\right|$.

\footnotetext{
${ }^{1}$ ef. Hardy, Littlewood and Polya, Inequalities (Cambridge, 1934), p. 34.
} 
Since $f$ is differentiable, it is possible to choose $\delta>0$ so small that, if $\left|x_{i}\right|<\delta$, then $\left|y_{i}\right|<\epsilon^{\prime}\left|x_{i}\right|$ where $\epsilon^{\prime}=\epsilon /(n+1)$. In this case we have from (5),

Thus the necessity is proved.

$$
\begin{aligned}
\left|q_{i} y_{i}\right| \leqslant \epsilon^{\prime}\left|x_{1}\right| \ldots\left|x_{n+1}\right| \text { so, from (4) } & \\
\left|\mathrm{D}\left(f ; x_{1}, \ldots, x_{n+1}\right)\right| & \leqslant \Sigma\left|q_{i} y_{i}\right| \\
& \leqslant(n+1) \epsilon^{\prime}\left|x_{1}\right| \ldots\left|x_{n+1}\right| \\
& =\epsilon\left|x_{1}\right| \ldots\left|x_{n+1}\right| .
\end{aligned}
$$

\section{B. Sufficiency.}

Let $u_{i}$ be the unit vector with $i^{\text {th }}$ co-ordinate unity, and all the others zero. Let $x=\left(x^{1}, \ldots, x^{n}\right)$ be any vector, so that

$$
x=x^{1} u_{1}+\ldots+x^{n} u_{n} \text {. }
$$

As before let $\epsilon>0, \epsilon^{\prime}=\epsilon /(n+1)$.

Let $x, y$ be any two vectors, none of the components of $y$ being zero. Let $\Psi(x, y)=D\left(f ; x, y^{1} u_{1}, \ldots, y^{n} u_{n}\right) /\left(y^{1} y^{2} \ldots y^{n}\right)$.

The determinant may be evaluated by subtracting $x^{i} / y^{i}$ times row $(i+1)$ from row 1 , for $i=1, \ldots, n$ and expanding in terms of the first column. Its value is seen to be

$$
\Psi(x, y)=f(x)-\sum_{i=1}^{n} \frac{x^{i}}{y^{i}} f\left(y^{i} u_{i}\right) .
$$

Thus there exists $\delta>0$ such that if $|x|,|y|<\delta$,

$$
|\Psi(x, y)|<\epsilon^{\prime}|x| \text {. }
$$

Now let $t, t^{\prime}$ be two non-zero real numbers, each less than $\delta$ in absolute value. Apply the above formula with $x=t u_{1}, y=t^{\prime} u_{1}+z$, where the first component of $z$ vanishes (though no other does) and $|z|$ is so. small that $|y|<\delta$. We obtain the result that

$$
\text { if }|t|,\left|t^{\prime}\right|<\delta, \quad\left|\frac{f\left(t u_{1}\right)}{t}-\frac{f\left(t^{\prime} u_{1}\right)}{t^{\prime}}\right|<\epsilon^{\prime} .
$$

Since $\epsilon$ can be chosen arbitrarily, the limit $\lim _{t \rightarrow 0} \frac{f\left(t u_{1}\right)}{t}$ exists. If the value of this limit is $p_{1}$, we find, keeping $\epsilon$ fixed and letting $t^{\prime} \rightarrow 0$. in (7), that

$$
\left|f\left(t u_{1}\right)-p_{1} t\right| \leqslant \epsilon^{\prime}|t| \text {. }
$$

By a similar argument, numbers $p_{2}, \ldots, p_{n}$ exist such that

$$
\left|f\left(t u_{i}\right)-p_{i} t\right| \leqslant \epsilon^{\prime}|t|
$$

provided that $|t|<\delta$.

Now let $|x|<\delta$ so that $\left|x^{i}\right|<\delta(i=1, \ldots, n)$. 
Then, by (6), (8),

$$
\begin{aligned}
& \left|f(x)-\sum_{i=1}^{n} p_{i} x^{i}\right| \leqslant\left|f(x)-\sum_{i=1}^{n} f\left(x^{i} u_{i}\right)\right|+\sum_{i=1}^{n}\left|f\left(x^{i} u_{i}\right)-p_{i} x^{i}\right| \\
& =|\Psi(x, x)|+\sum_{i=1}^{n}\left|f\left(x^{i} u_{i}\right)-p_{i} x^{i}\right| \\
& <(n+1) \epsilon^{\prime}=\epsilon .
\end{aligned}
$$

Thus $f(x)$ is differentiable.

\section{Quene's College,}

DUNDEE. 